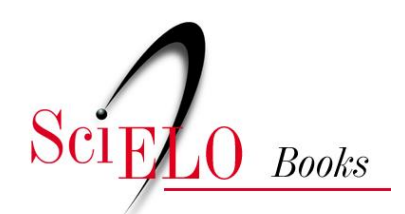

\title{
Experiencias de buenas prácticas 13. ¡Mis manos enseñan, tus ojos aprenden! Fotografía y producción de video en la educación de personas sordas
}

\author{
Jaime Sarmiento
}

\section{SciELO Books / SciELO Livros / SciELO Libros}

SARMIENTO, J. ¡Mis manos enseñan, tus ojos aprenden! Fotografía y producción de video en la educación de personas sordas. In: GALLEGOS NAVAS, M., ed. La inclusión de las TIC en la educación de personas con discapacidad: relatos de experiencias [online]. Quito: Editorial AbyaYala, 2019, pp. 171-181. ISBN: 978-9978-10-495-8. https://doi.org/10.7476/9789978104958.0016.

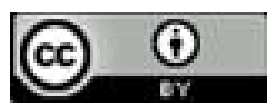

All the contents of this work, except where otherwise noted, is licensed under a Creative Commons Attribution 4.0 International license.

Todo o conteúdo deste trabalho, exceto quando houver ressalva, é publicado sob a licença Creative Commons Atribição 4.0.

Todo el contenido de esta obra, excepto donde se indique lo contrario, está bajo licencia de la licencia $\underline{\text { Creative }}$ Commons Reconocimento 4.0. 


\section{3. iMis manos enseñan, tus ojos aprenden! Fotografía y producción de video en la educación de personas sordas ${ }^{47}$}

"La primera tarea de la educación es agitar la vida, pero dejarla libre para que se desarrolle”

(María Montessori)

Fe y Alegría Ecuador

Ecuador

Quito

Responsable: Jaime Sarmiento

Gestores de la práctica: Para la realización de la práctica se contó con la participación de las siguientes personas: Estudiantes sordos: Damaris Lituma, Karen Mora y Yulissa Veintimilla (Presentadoras), Micaela Pazmiño, Evelyn Ordoñez, Anderson Franco y Rosario Álvarez (Realización), Paola Acosta y Daniel Navarrete (Camarógrafos), William López (Mimo) y Jonathan Araujo (Postproducción), 11 estudiantes con discapacidad auditiva, Docentes de la Unidad de Educación Especial Fe y Alegría de Santo Domingo,( Jaime Sarmiento, coordinador de proyecto, Leonardo Llumiquinga, facilitador del proceso de formación y asesor especialista en producción audiovisual) y con la participación de José Pico, coordinador del equipo y facilitador del proceso. Génesis Limaico, Silvia Núñez, Soledad Montalván, Pablo Quezada y Jonathan Zambrano.

El uso de tecnología en la dinámica cotidiana del aula (radio, televisión, internet), permite la construcción de la opinión autónoma de los estudiantes sordos, convirtiéndolos en agentes participativos y responsables del proceso de aprendizaje.

Esta experiencia muestra con claridad la inclusión del carácter lúdico en la experiencia de aprendizaje y el respeto a las diversas formas de comunicación de los estudiantes, lo que permite que se establezca un diálogo intercultural cualificando y mejorando los aprendizajes del conjunto de actores comprometidos (docentes familias y estudiantes). Esta experiencia se constituye en ejemplo de emprendimiento en la que los conocimientos del aula se transfieren en beneficio de la comunidad.

47 Para poner énfasis en un lenguaje positivo y adecuado al referirse a las personas con discapacidad, es necesario resaltar que las personas sordas tienen una lengua propia y pueden comunicarse, por tanto no es correcto referirse a las personas sordas como sordomudas, son simplemente sordas. 
172

\section{Motivación}

A partir de un contexto de exclusión, de una oferta educativa bajo un enfoque clínico que históricamente ha limitado el desarrollo integral de las personas sordas y de una serie de condiciones que han limitado la práctica de los derechos lingüísticos y comunicaciones de las personas sordas, la práctica educativa de Fe y Alegría, desarrollada desde un enfoque bilingüe bicultural, partiendo de un proceso de formación, busca diseñar e implementar un producto educativo y comunicacional que permita abrir a la sociedad en general, una ventana al mundo de las personas sordas con el apoyo de las TIC. El proceso de formación se convirtió en un ejercicio de transferencia de conocimientos para que sean los estudiantes sordos quienes creen la propuesta de comunicación, y a partir de ella, difundan su lengua y la forma en que ven y viven sus problemas, sueños e intereses. Entre otros motivos, esta propuesta surgió porque: ${ }^{48}$

- Existe una limitada oferta de los medios tradicionales de comunicación para la población sorda. La oferta se limita a la interpretación en lengua de señas (realizada por intérpretes oyentes) de algunas noticias, en ciertos canales de televisión.

48 Véase también: Asociación Fe y Alegría Ecuador. (2013). La Educación Especial en Fe y Alegría Ecuador. Quito: Fe y Alegría. Asociación Fe y Alegría Ecuador. (2014). Proyecto Mejora de la Calidad Educativa e Inclusión de estudiantes con discapacidad auditiva con la participación de familias, escuela y comunidad. Quito: Fe y Alegría. Asociación Fe y Alegría Ecuador. (2015). Guía para la Inclusión de Estudiantes Sordos en la Educación Regular. Quito: Fe y Alegría. Asociación Fe y Alegría Ecuador. (2016). Análisis de las fortalezas, retos y prácticas alineadas al Modelo Bilingüe Bicultural de 5 instituciones para estudiantes sordos. Informe de Análisis y Recolección de Información. Quito: Fe y Alegría. CONADIS. (2013). Agenda Nacional para la Igualdad en Discapacidades 2013-2017. Quito: Consejo Nacional de Igualdad de Discapacidades. INEC. (2010). Población por condición de discapacidad, según provincia, cantón, parroquia y área de empadronamiento. Quito: Instituto Nacional de Estadísticas y Censos. 


\section{3}

- No existe la presencia de la persona sorda en los medios, no se evidencian sus problemas, necesidades, expresiones o sueños. Cuando temas relacionados con las personas sordas aparecen en los medios tradicionales, éstos se limitan a la interpretación de los oyentes sobre la realidad de la persona sorda.

- Existe una limitada producción de información, notas, material educativo, contenidos artísticos o comunicacionales desde la comunidad sorda. En los medios tradicionales esta producción es prácticamente nula y en medios alternativos, como sitios web para compartir videos, la producción que aparece se limita a informar de temas de actualidad. Adicionalmente, esta producción carece de un trabajo profesional y de la presencia de jóvenes sordos.

- Hay una demanda limitada por parte de la comunidad sorda, que si bien exige su derecho a la libre expresión y al acceso a la información, por un lado se limita a exigir lo que ya hacen los medios tradicionales, como es la interpretación en lengua de señas de las noticias, y por otro lado no hace uso de las herramientas disponibles para producir información desde medios alternativos.

- Por otro lado, se evidencia un problema de baja autoestima, inseguridad y retraimiento que si bien puede ser una condición común en todo adolescente o joven en algunos contextos, se profundiza en adolescentes sordos, producto de la exclusión y discriminación que afectan a esta población. Esto hace muy difícil que un adolescente se enfrente a una cámara y a la exposición pública. Se requiere un trabajo sostenido en el desarrollo de su identidad como persona sorda y en la autovaloración para vencer los temores y afrontar nuevos retos, como el que plantea la propuesta de comunicación desarrollada por Fe y Alegría. 
174

Figura 90

Trabajo sostenido en Fe y Alegría

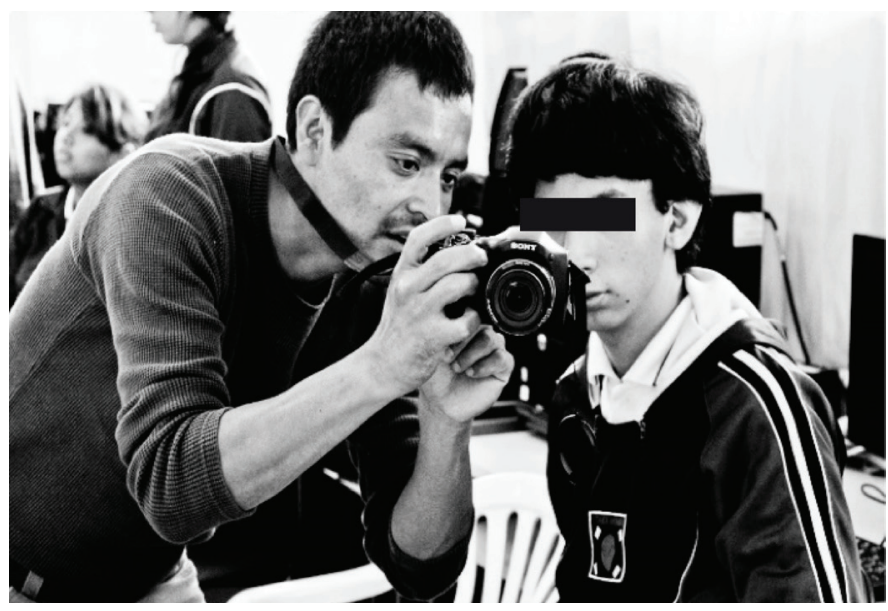

Foto: Jaime Sarmiento (2017)

\section{Objetivos}

\section{Objetivo general}

Implementar una propuesta educativa, comunicacional y de sensibilización sobre los derechos de las personas sordas con el apoyo de las tecnologías de la información y comunicación, en donde estudiantes sordos ejecuten todas las fases de pre y post producción de los contenidos.

\section{Objetivos específicos}

- Alentar una oferta informativa inclusiva en los medios de comunicación y diversificar la producción de información, notas, contenidos artísticos y comunicacionales desde la comunidad sorda.

- Contribuir al ejercicio de la libertad de expresión y al acceso a información de las personas sordas en Ecuador. 
- Articular la propuesta al proceso educativo de estudiantes sordos, fortaleciendo sus capacidades en el uso de las TIC y en la lectoescritura.

\section{Desarrollo de la experiencia}

Ante la exclusión y subvaloración del rol de las personas con discapacidad, muchas personas y organizaciones han optado por usar las tecnologías de la información y comunicación (en adelante TIC) para llenar el vacío de contenidos o para difundir información relativa a los intereses de las personas sordas.

Siguiendo el ejemplo de la comunidad sorda, a inicios del 2013, Fe y Alegría Ecuador ${ }^{49}$ se planteó desarrollar una propuesta de comunicación para la promoción de los derechos de las personas sordas, mediante el uso de las TIC. Esta propuesta se viene desarrollando en la Unidad de Educación Especial Fe y Alegría (En adelante UEEFA) de la ciudad de Santo Domingo. La UEEFA se creó en 1984 y es la única institución de educación especial de la Provincia de Santo Domingo de los Tsáchilas. En esta institución se ofrecen servicios de estimulación temprana, rehabilitación física, terapia de lenguaje, educación inicial y educación general básica a 150 niñas, niños, adolescentes y jóvenes con discapacidad auditiva e intelectual.

Formación en fotografía. Para la materialización de esta propuesta de comunicación, se inició con un programa especial de formación en fotografía. Por medio de este proceso de formación se logró usar

49 Fe y Alegría es una institución de carácter privado, sin fines de lucro, que brinda servicios de educación y promoción social con énfasis en los sectores y grupos poblacionales tradicionalmente excluidos. Está presente en 14 provincias de Ecuador, principalmente en áreas urbano-marginales y rurales donde atiende las necesidades de educación de más de 29000 niños y jóvenes, a través de sus 77 centros y servicios educativos. Su oferta abarca la educación formal con servicios de educación inicial, educación general básica, bachillerato, educación especial, así como servicios de educación no formal. 
la fotografía como una herramienta de comunicación para transmitir los puntos de vista personales y la percepción del contexto que rodea a jóvenes sordos de la ciudad de Santo Domingo. Luego del proceso de formación se seleccionó una muestra fotográfica, misma que se ha presentado en varios espacios, encuentros y eventos para promocionar los derechos de las personas sordas, como por ejemplo en la Semana Internacional de la Persona Sorda. ${ }^{50}$ De este proceso educativo surgió la exposición "Fotografía para la Inclusión” la cual reúne una serie de fotografías de un grupo de estudiantes sordos de Fe y Alegría. Esta muestra fotográfica permitió apreciar el talento particular de estudiantes sordos, su especial sensibilidad y su potencial en el campo de la fotografía.

Figura 91

Formación en fotografía en Fe y Alegría

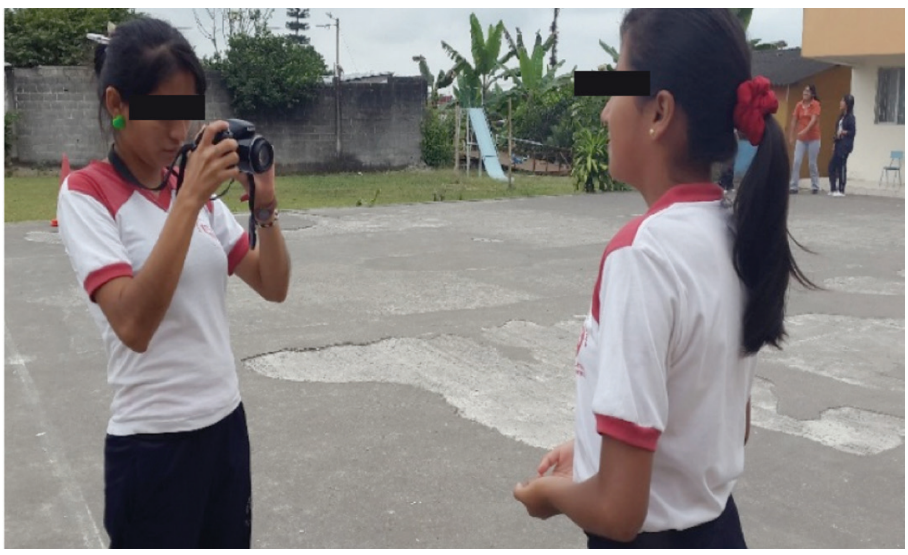

Foto: Jaime Sarmiento (2017)

Formación en producción audiovisual. Posteriormente, se desarrolló un proceso de formación en producción audiovisual, dirigido a un

50 La Semana Internacional de las Personas Sordas comenzó a celebrarse el 28 de septiembre de 1958 para conmemorar el primer Congreso Mundial de la Federación Mundial de Personas Sordas que se celebró en septiembre de 1951, por lo que se celebra durante la última semana completa de septiembre. 
grupo de 11 jóvenes sordos y 5 docentes de la UEEFA. Durante la realización de este programa de formación se concretó la producción de la revista televisiva para sordos y oyentes denominada "Mis manos enseñan, tus ojos aprenden". El producto del proceso de formación fue la edición y publicación en Internet de la revista televisiva, bajo una metodología activa de formación. En el proceso de construcción de la propuesta, se definió que la temática a abordar en la revista, partiría de las vivencias y distintas visiones del entono social que tiene la juventud sorda, con sus personajes, sus temas de interés y en continuo intercambio con otros actores institucionales y sociales. La revista se definió así como una producción educativa y de comunicación diseñada, filmada y editada por estudiantes sordos, con el apoyo de docentes de la UEEFA y la asesoría de Leonardo Llumiquinga, consultor experto en producción audiovisual.

\section{Figura 92}

Formación en producción audiovisual en Fe y Alegría

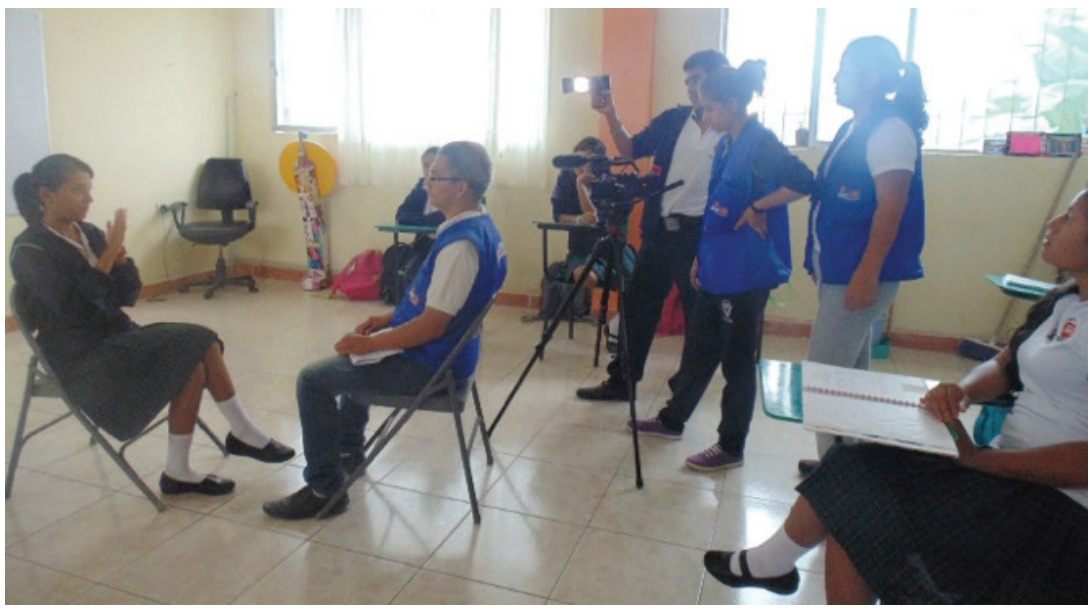

Foto: Jaime Sarmiento (2017)

La metodología desarrollada, como se resume en la Figura 101, consistió en: 
- Formación teórico-práctica sobre producción audiovisual.

- Acompañamiento y seguimiento.

- Publicación de la revista en Internet.

- Difusión de la revista en eventos y redes sociales.

Figura 93

Metodología de la propuesta

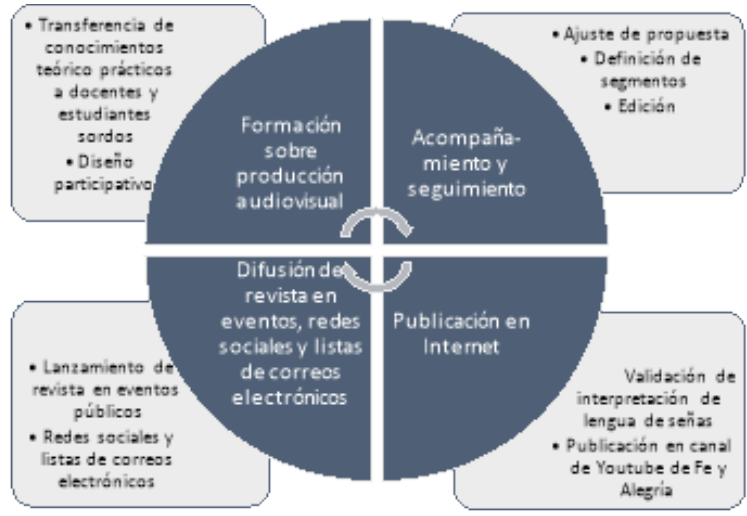

Fuente: Jaime Sarmiento (2017)

Para la elaboración de este producto comunicacional y educativo, se inició con un taller sobre producción audiovisual con una duración de dos meses, en sesiones de trabajo consecutivo, de cuatro días por semana y 4 horas diarias de formación. Esta fase de transferencia de conocimientos estuvo a cargo de Leonardo Llumiquinga, consultor especialista en producción audiovisual y en formación en el ámbito de la comunicación y uso de equipos de filmación, edición y TIC para la comunicación social..$^{51}$

La formación inició con la revisión del marco teórico sobre la organización del equipo de trabajo, la labor del equipo de dirección y del equipo de producción, formato y género de programas audiovisuales, es-

51 El consultor especialista en TIC y comunicación social, Leonardo Llumiquinga preparó todos los contenidos, tutoriales y recursos para este programa de formación. 
tructura narrativa y recursos narrativos. Para esta primera fase se utilizaron tutoriales digitales y métodos activos para llevar la teoría a la práctica, para conocer el manejo de una cámara profesional de video y para crear en equipo la propuesta de comunicación.. En el desarrollo de los talleres, se definió que la revista audiovisual saldría al aire de forma periódica, pero sujeta a la dinámica de la UEEFA y a las facilidades para la edición del material grabado. El programa de formación inició con un grupo de docentes para asegurar la permanencia de los conocimientos y contenidos y para facilitar la incorporación de esta propuesta en la malla curricular de la UEEFA. Esta primera instancia sirvió además para fortalecer el monitoreo a jóvenes sordos en el taller de producción audiovisual subsiguiente.

La segunda etapa de la propuesta consistió en la formación a jóvenes sordos, en donde se combinó la teoría y la práctica en producción audiovisual, con narrativa cinematográfica y con el apoyo de la lengua de señas ecuatoriana. Finalmente, se trabajó con jóvenes y docentes, en la producción de la revista audiovisual hasta ubicarla en un sitio web de reproducción de videos. De esta manera, la experticia surgida en la realización de estos insumos se concatenó, facilitando los procesos y el sostenimiento en la producción para realizar una revista audiovisual de contenido y con estándares de calidad.

Es necesario resaltar que durante todo el proceso de producción, mediante una metodología de lluvia de ideas, junto a los estudiantes se fueron creando tanto la estructura narrativa del programa, como los reportajes y las temáticas a tratar. Incluso el nombre de la revista surgió de una de las estudiantes sordas. Fue así que la revista pasó a llamarse "Mis manos enseñan, tus ojos aprenden", título que revela la intencionalidad de la propuesta, la interacción entre el mundo sordo y oyente, la relevancia de la lengua de señas y el enfoque educativo de la propuesta.

\section{Logros obtenidos}

La revista "Mis manos enseñan, tus ojos aprenden", está articulada al proceso educativo de jóvenes sordos, potenciando las capacidades 
para la redacción, la planificación, el trabajo en equipo, el uso de las TIC y la promoción de los derechos de comunicación, derechos lingüísticos, libertad de expresión e identidad de las personas sordas.

Una vez finalizada la primera edición, la revista se definió como un producto educativo, comunicacional, inclusivo y de innovación social, que muestra de qué forma se pueden desarrollar contenidos inclusivos en donde oyentes y sordos compartan y aprendan juntos, más allá de las barreras y limitaciones de los medios tradicionales de comunicación y de los estigmas sociales. Adicionalmente, se convirtió en una muestra del potencial que tienen jóvenes sordos para producir contenidos de calidad que pueden ser aprovechados como material educativo desde un enfoque bilingüe bicultural.

La revista audiovisual abre las puertas al mundo de las personas sordas, refuerza la identidad de la persona sorda mediante la preeminencia de la lengua de señas ecuatoriana (En adelante LSEC) por sobre la comunicación oral y busca posicionar el protagonismo de jóvenes sordos, sin olvidar el diálogo intergeneracional e intercultural con el mundo de los oyentes.

Producto de esta experiencia, actualmente se cuenta con una metodología de formación en producción audiovisual para estudiantes sordos, cuatro revistas audiovisuales producidas por estudiantes sordos y cinco mini documentales sobre una propuesta de mejora de la calidad de la educación para personas sordas. Adicionalmente, se cuenta con un equipo de tres docentes y un equipo de once estudiantes sordos formados en producción audiovisual.

Tras la difusión de las revistas en el canal de YouTube de Fe y Alegría y en eventos de lanzamiento realizados en la ciudad de Santo Domingo, se ha alcanzado una importante difusión de la misma, alcanzando rápidamente más de 3000 visitas luego de su publicación.

La realización de la revista ha creado nuevas expectativas y proyecciones profesionales en los participantes, ligadas al campo de la producción 
audiovisual, el periodismo y la fotografía. Esto se ha evidenciado a través del seguimiento de la coordinación, al equipo de producción de la revista.

Finalmente, se cuenta con cuatro revistas audiovisuales ${ }^{52}$ producidas por estudiantes sordos, con el apoyo de sus docentes, como material de sensibilización, educación y promoción de la lengua de señas y de los derechos de las personas sordas.

\section{La teoría del Neuroaprendizaje a través de las TIC en personas que asisten al servicio de rehabilitación}

"El primer movimiento de la pequeña mano hacia las cosas, el impulso de este movimiento, representa el esfuerzo del yo por penetrar en el mundo"

(María Montessori)

Centro de Rehabilitación

Ecuador

Quito

Responsable: Juan Carlos Guachamín

Gestores de la práctica: Esta experiencia se desarrolló con los miembros del equipo de rehabilitación (terapista físico, terapista del lenguaje).

Esta buena práctica gira en torno a actividades cotidianas desarrolladas a través de diferentes software los cuales aplicados de acuerdo a los intereses y necesidades de cada niño.

Se desarrollaron con una metodología altamente participativa, los profesionales utilizaron un gran abanico de posibilidades de estrategias didácticas para trabajar en varias áreas del conocimiento como pre matemática, funciones básicas, lenguaje, vocabulario, comprensión lectora.

La presente experiencia permite maximizar los resultados de la rehabilitación de los niños, niñas con trastornos neurológicos. Mediante el trabajo colaborativo con el equipo multimodal de la institución conformado por los profesionales en Terapia Física, Terapia de Lenguaje y Psicología Clínica. El entrenamiento neurocognitivo mediado por las TIC permite la modificación de las funciones cerebrales de manera temporal o permanente, así como de la conducta de los niños y niñas con trastornos neurológicos.

52 Estas revistas están disponibles a través del enlace al canal de YouTube de Fe y Alegría: https://goo.gl/qF2ynm 